\title{
Sweep-Stick Mechanism of Heavy Particle Clustering in Fluid Turbulence
}

\author{
Susumu Goto \\ Department of Mechanical Engineering and Science, Kyoto University, Yoshida-Honmachi, Sakyo, Kyoto 606-8501, Japan \\ J. C. Vassilicos \\ Department of Aeronautics, Imperial College, London, SW7 2AZ, United Kingdom \\ Institute for Mathematical Sciences, Imperial College, London, SW7 2PE, United Kingdom
}

(Received 11 July 2007; published 8 February 2008)

\begin{abstract}
It is proposed that the inertial range clustering of small heavy particles in fluid turbulence occurs as a result of the sweep-stick mechanism which causes inertial particles to cluster so as to mimic the clusters of points where the fluid acceleration is perpendicular to the direction of highest contraction between neighboring particles. Direct numerical simulations of inertial particles subjected to linear Stokes drag and suspended in homogeneous isotropic turbulence support the validity of the sweep and stick properties on which the sweep-stick mechanism is based, and also support the clustering consequences of this mechanism. It also explains the observed Stokes-number dependence of inertial particle clustering.
\end{abstract}

PACS numbers: 47.27.- $\mathrm{i}, 47.10 .-\mathrm{g}$

Turbulence spreads suspended impurities, but it can also bring them together to form clusters. For example, bubbles, which are much lighter than the surrounding fluid, may be used to visualize eddies (see, e.g., [1]), since they tend to accumulate along the center line of swirls. It is also well known [2-4] that particles heavier than the surrounding fluid cluster in turbulence. This phenomenon, sometimes called preferential concentration, is crucial in various processes where the particle collision rate is a significant factor (such as combustion or chemical reaction in turbulence, raindrop growth in clouds, planet formation in the early solar system, etc.). Thus, it has been studied extensively in many areas of physics and mechanics.

In turbulence at a relatively low Reynolds number, the heavy particle clustering is due to the action of the smallest-scale (i.e. the Kolmogorov length $\eta$ ) eddies. Specifically, heavy particles are centrifuged out of the coherent eddies, and accumulate in low-vorticity (highstrain-rate) regions. However, this picture is not valid for developed turbulence at a higher Reynolds number, and the clustering is not a single-scale phenomenon but has a multiscale nature [5-7]. This is because not only the smallest-scale but also multiscale coherent eddies in developed turbulence play a role in the preferential concentration, and therefore clusters differ significantly in space from the locations of low-vorticity regions.

On the other hand, it has been pointed out [7-11] that the clusterings of heavy particles and fluid acceleration are strongly related. Especially, Refs. [7,9] claim that the spatial distribution of heavy particles reflects that of the stagnation points of the fluid acceleration in twodimensional inverse-energy cascading turbulence. Here, we extend this picture to three-dimensional turbulence by extending and refining the sweep-stick mechanism proposed (but not named) in [7,9] to three-dimensional turbulence.
In what follows, we deal with the clustering of heavy particles suspended in statistically stationary homogeneous isotropic turbulence (HIT), $\boldsymbol{u}(\boldsymbol{x}, t)$. We assume that only the Stokes drag drives the motion of the particles, and the equation of motion for a particle (the velocity and the position vectors of which are denoted by $\boldsymbol{v}_{p}$ and $\boldsymbol{x}_{p}$, respectively) is expressed, neglecting gravity, by

$$
\frac{d}{d t} \boldsymbol{v}_{p}=-\frac{1}{\tau_{p}}\left(\boldsymbol{v}_{p}(t)-\boldsymbol{u}\left(\boldsymbol{x}_{p}(t), t\right)\right)
$$

This assumption is justified (see Ref. [12] for a detailed discussion based on first principles) when the radius $r$ of the particles is much smaller than $\eta$; the Reynolds number based on $r$ and the relative velocity between the particle and the surrounding fluid is small enough for the Stokes approximation of the flow around the particle to be valid; and the mass density of the particle is much greater than the fluid density. The Stokes drag relaxes the particle velocity $\boldsymbol{v}_{p}$ towards the fluid velocity at $\boldsymbol{x}_{p}$ within a time scale $\tau_{p}$. The normalised relaxation time $\mathrm{St}=\tau_{p} / \tau_{\eta}$, where $\tau_{\eta}$ is the Kolmogorov microscale, is called the Stokes number.

For simplicity, interactions between particles and feedback onto fluid motion from the particles are neglected. These might be treated as secondary effects for the clustering of particles which is created by the primary effect shown below.

The sweep-stick mechanism of particle clustering is described as follows. First, the fluid acceleration field $\boldsymbol{a}(=$ $\partial \boldsymbol{u} / \partial t+\boldsymbol{u} \cdot \nabla \boldsymbol{u})$ is swept by the local fluid velocity $\boldsymbol{u}$. This has been demonstrated $[9,13]$ for points where $\boldsymbol{a}=\mathbf{0}$ or where $|\boldsymbol{a}| \ll a^{\prime}$ (where $a^{\prime}$ is the rms value of an acceleration component) in two-dimensional inverse-energy cascading HIT. Second, particles tend to stick to and move with points where $\boldsymbol{a}=\mathbf{0}$ because 


$$
\boldsymbol{v}_{p} \approx \boldsymbol{u}\left(\boldsymbol{x}_{p}, t\right)-\tau_{p} \boldsymbol{a}\left(\boldsymbol{x}_{p}, t\right),
$$

which follows from (1) when St is small [2]. As a result, particles on $\boldsymbol{a}=\mathbf{0}$ points move together with these points with velocity $\boldsymbol{u}$, whereas particles on points where $\boldsymbol{a} \neq \mathbf{0}$ move away from these points with relative velocity $\tau_{p} \boldsymbol{a}$. This is the basis of the sweep-stick mechanism, and it is broadly sufficient to explain particle clustering and its coincidence with $\boldsymbol{a}=\mathbf{0}$ clusters in two-dimensional inverse-energy cascading HIT because $\boldsymbol{a}=\mathbf{0}$ points abound in such flows. However, whereas particle clustering is observed in our DNS of three-dimensional HIT (Fig. 1, below), $\boldsymbol{a}=\mathbf{0}$ points do not correlate well with this clustering. In fact, these points turn out to be very rare [14] and, as we explain below, not the stickiest either. The stickiness requires the particle compressibility to be properly defined.

It has been noted by various previous authors (starting with [2]) that $\nabla \cdot \boldsymbol{v}_{p} \approx-\tau_{p} \nabla \cdot \boldsymbol{a}$, as a result of (2), and that this implies that particles converge towards each other where and when $\nabla \cdot \boldsymbol{a}>0$. More specifically, this convergence can in fact be expected to occur predominantly along the direction parallel to the eigenvector $\boldsymbol{e}_{1}$ corresponding to the largest (positive) eigenvalue of the symmetric part of the acceleration gradient tensor $\nabla \boldsymbol{a}$. The three eigenvectors of this symmetric tensor are denoted by $\boldsymbol{e}_{i}(i=1,2,3)$.

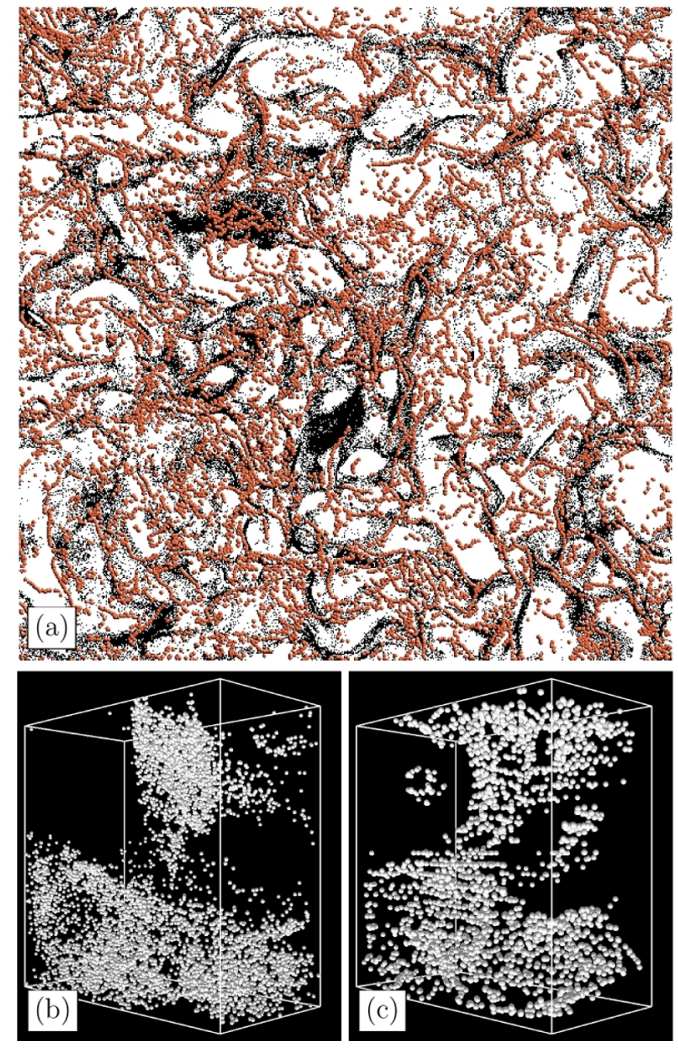

FIG. 1 (color online). (a) Heavy small particles (black small dots) of $\mathrm{St}=2$ in a thin layer (thickness $5 \eta$ and side length $500 \eta$ ), and the set $\mathcal{A}$ of points where (3) is satisfied (red larger balls). The three-dimensional view is shown in (b) for the particles and in (c) for $\mathcal{A}$ in a box of size $50 \eta \times 50 \eta \times 25 \eta$.
Instead of $\boldsymbol{a}=\mathbf{0}$ points, consider the three surfaces defined by $\boldsymbol{a} \cdot \boldsymbol{e}_{i}=0$ for $i=1,2$, 3. If local points on these surfaces move, predominantly, with the local fluid velocity $\boldsymbol{u}$ (sweep), then particles on such points may move with these points in all $\boldsymbol{e}_{i}$ directions (first part of stick) by virtue of $\left(\boldsymbol{v}_{p}-\boldsymbol{u}\right) \cdot \boldsymbol{e}_{i} \approx-\tau_{p} \boldsymbol{a} \cdot \boldsymbol{e}_{i}=0$. However, particles will not necessarily converge together except along $\boldsymbol{e}_{1}$ when $\lambda_{1}>0$. Hence, only in this direction $\boldsymbol{e}_{1}$ is the stick side of the mechanism effective (second part of stick). Particles in the neighborhood of the surfaces

$$
\boldsymbol{e}_{1} \cdot \boldsymbol{a}=0 \text { and } \lambda_{1}>0
$$

are therefore compressed together and will stick on these surfaces because, due to the first part of stick, they move together with them in the direction $\boldsymbol{e}_{1}$. Indeed, whereas particles and points on these surfaces may diverge in the other directions $\boldsymbol{e}_{2}$ and $\boldsymbol{e}_{3}$, the compressibility in the $\boldsymbol{e}_{1}$ direction will bring the particles back onto other points of the surface defined by (3), thus enforcing the stick side of the mechanism as the particle and the surface move together in that direction. Points where (3) holds abound and we denote $\mathcal{A}$ the set of such points.

In order to numerically verify the proposed mechanism (see Fig. 2 for sweep and Fig. 3 for stick) of the particle clustering and its prediction (see Figs. 1 and 4), we simulate the motion of $256^{3}$ particles suspended in statistically stationary turbulence (the Taylor-length based Reynolds number $R_{\lambda} \approx 187$ ) in a periodic cube. The resolution of the velocity field is $512^{3}$ grid points, and one direction of the periodic box fits about 7 integral lengths $\mathcal{L}$. The integral time $u^{\prime} / \mathcal{L}$ is about $25 \tau_{\eta}\left(u^{\prime}\right.$ is the rms value of a fluid velocity component). The positions of the particles are numerically simulated by integrating (1) together with the Navier-Stokes equations for the velocity field of an incompressible fluid by the 4th-order Runge-Kutta method and the Fourier spectral method.

A direct numerical evidence of the sweep-stick mechanism is given in Fig. 1. In (a), we plot the spatial distribution of particles of $\mathrm{St}=2$ (black small dots) in a thin (thickness $5 \eta$ ) layer, together with the distribution of the

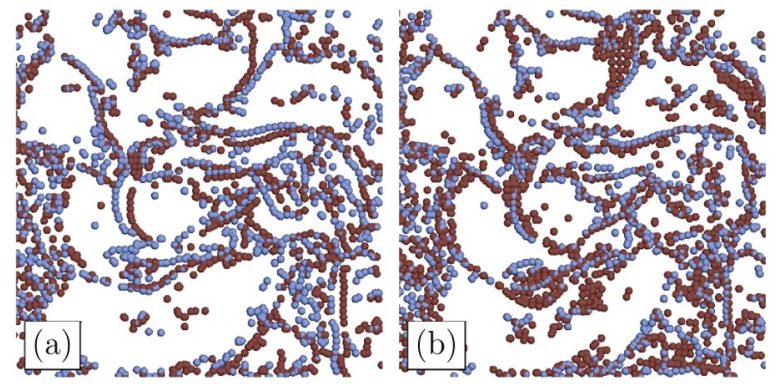

FIG. 2 (color online). (a) Distributions of points in $\mathcal{A}$ at two different times $t$ (dark red points) and $t+\delta t$ (light blue) on a plane of size $(140 \eta)^{2}$. (b) $\mathcal{A}$ at $t+\delta t$ (light blue) and the structure (dark red) of $\mathcal{A}$ at $t$ swept by the local fluid velocities for the duration $\delta t$. 


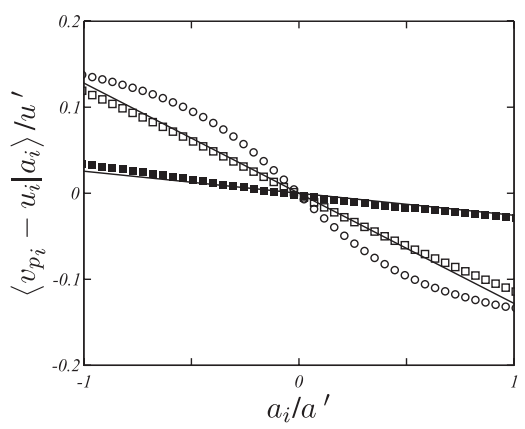

FIG. 3. Average relative velocity component between a particle and the fluid at the particle position $\boldsymbol{x}_{p}$ conditioned by the fluid acceleration component at $\boldsymbol{x}_{p}$. Normalized by the rms values $u^{\prime}$ and $a^{\prime}$ of fluid velocity and acceleration. $\mathbf{\square}, \mathrm{St}=$ $0.1 ; \square, 0.5 ; \circ, 2$. Formula (2) for $\mathrm{St}=0.1$ and 0.5 is indicated by the solid lines.

set $\mathcal{A}$ of points (red balls) which satisfy (3) on the central plane of the thin layer. $\mathcal{A}$ has been identified by the Newton-Raphson method, and $\boldsymbol{a}$ is calculated from its definition, $\partial \boldsymbol{u} / \partial t+\boldsymbol{u} \cdot \nabla \boldsymbol{u}$. The two distributions coincide quite well. This coincidence can be observed in any arbitrarily chosen layer. Indeed, we show in Figs. 1(b) and 1(c) the three-dimensional spatial distribution of the particles and the set $\mathcal{A}$, respectively, in a box of size $50 \eta \times 50 \eta \times$ $25 \eta$. A good coincidence is observed again.

Now, we discuss numerical verifications of each of the sweep and stick sides of our mechanism. Concerning the sweep of surfaces where $\boldsymbol{e}_{1} \cdot \boldsymbol{a}=0$, we plot $\mathcal{A}$ at two different times, $t$ and $t+\delta t$ (here $\delta t=0.5 \tau_{\eta}$ ), in Fig. 2(a). It is observed that $\mathcal{A}$ moves while keeping its coherence. In Fig. 2(b), we plot $\mathcal{A}(t+\delta t)$ and the structure which results at $t+\delta t$ from simply sweeping $\mathcal{A}(t)$ by the local fluid velocity $\boldsymbol{u}$. The good coincidence of $\mathcal{A}(t+\delta t)$ with this swept structure supports the sweep side of our mechanism. We emphasize that this coincidence is not trivial; other properties, such as constant-velocity points [15] and constant-velocity-component surfaces, are not typically swept by the velocity field as we have confirmed by the same time stepping method.

Concerning the stick side of our mechanism, we verify (2), which is really what our mechanism is based on. We plot in Fig. 3 the average value of the $i$ th component of $\boldsymbol{v}_{p}-\boldsymbol{u}$ conditioned by $a_{i}$ for $\mathrm{St}=0.1,0.5$ and 2. Recall that (2) is valid when $\tau_{p}$ is shorter than the typical time scale of turbulence. As expected, the formula is well satisfied for small $\tau_{p}$, say $\lesssim 0.5 \tau_{\eta}$, in the region $\left|a_{i}\right| \lesssim$ $a^{\prime}$. For $\tau_{p}$ larger than $\tau_{\eta}$, although the constant of proportionality is significantly larger than $-\tau_{p}$ (the slope is shallower), the relation that $v_{p i}-u_{i} \sim a_{i}$ is still well satisfied around $a_{i}=0$. It is only this proportionality (which leads to the feature that $\boldsymbol{a} \cdot \boldsymbol{e}_{1}=0$ surfaces are sticky for particles) that is essential in the argument of the sweep-stick mechanism.
A strong point of the sweep-stick mechanism is that it consistently explains the Stokes-number dependence of the clustering. It must be emphasized that the dependence cannot be explained solely in terms of the magnitude of the fluid acceleration, vorticity or strain rate.

Here, we briefly summarize the Stokes-number dependence of the particle clustering. The clustering is well understood in terms of the resonance between the coherent multiscale eddies and particles [6,7] as follows. (i) When $\tau_{p}$ is a few percent of $\tau_{\eta}$, only the smallest-scale eddies are resonant with the particles, and only the smallest-scale voids of particles are created. (ii) As $\tau_{p}$ increases and becomes larger than $\tau_{\eta}$, larger voids of particles appear because eddies larger than $\eta$ are resonant with the particles. The size of the voids increases with increasing $\tau_{p}$ until $\tau_{p}$ reaches the integral time (the longest time scale) of turbulence. (iii) On the other hand, as $\tau_{p}$ gets larger, smaller-scale voids of particles become faint because as $\tau_{p}$ increases, the resonant condition is violated from smaller-scale eddies.

A key to understanding this Stokes-number dependence in terms of the sweep-stick mechanism is the contraction rate $\gamma$ of particle blobs in the $e_{1}$ direction, $\gamma \approx \lambda_{1} \tau_{p}$. Here, we have used $\nabla \boldsymbol{v}_{p} \approx-\tau_{p} \nabla \boldsymbol{a}$. Since the particle velocity differs from the fluid velocity over time scales smaller than $\tau_{p}$, when $\tau_{p} \ll \gamma^{-1}$ the blob of particles contracts too slowly and many particles avoid much of the contraction as if they were fluid particles. In such a case, particle clusters become thick around the points defined by $\mathcal{A}$. On the other hand, when $\tau_{p} \gg \gamma^{-1}$ particle blobs contract quickly, but the particles are inert enough to overshoot the structure defined by $\mathcal{A}$. In such a case, the cluster structure becomes faint. Hence, in order for particles to stick best and form a sharp cluster with sufficient contraction in the $\boldsymbol{e}_{1}$ direction, $\tau_{p}$ must be comparable to $\gamma^{-1}$. Hence, only when

$$
\tau_{p} \approx \lambda_{1}^{-1 / 2}
$$

does the sweep-stick mechanism create sharp particle clusters. (Note that since the constant of proportionality in $v_{p i}-u_{i} \sim a_{i}$ is modified for larger $\tau_{p}$, (4) should also be modified accordingly in such a case.) The above argument implies that the case shown in Fig. 1 corresponds to a Stokes number for which the above condition is satisfied by many points in $\mathcal{A}$. We find that values taken by $\lambda_{1}$ in $\mathcal{A}$ range from 0 to $O\left(\tau_{\eta}^{-2}\right)$.

In order to verify the above mentioned Stokes-number dependence, we plot $\mathcal{A}$ and the spatial distribution of particles in a square of size $140 \eta \times 140 \eta(\mathrm{St}=2,0.1$ and 5) in Fig. 4. It is seen that the cluster of particles with $\mathrm{St}=2$ [Fig. 4(b)] is sharp and mimics $\mathcal{A}$ [the whole set shown in Fig. 4(a)]. If we decrease the Stokes number [see Fig. 4(c) for $\mathrm{St}=0.1$ ], the cluster thickens. Consequently, the many voids of particles observed in (b) for a larger St are filled with particles, and only small 


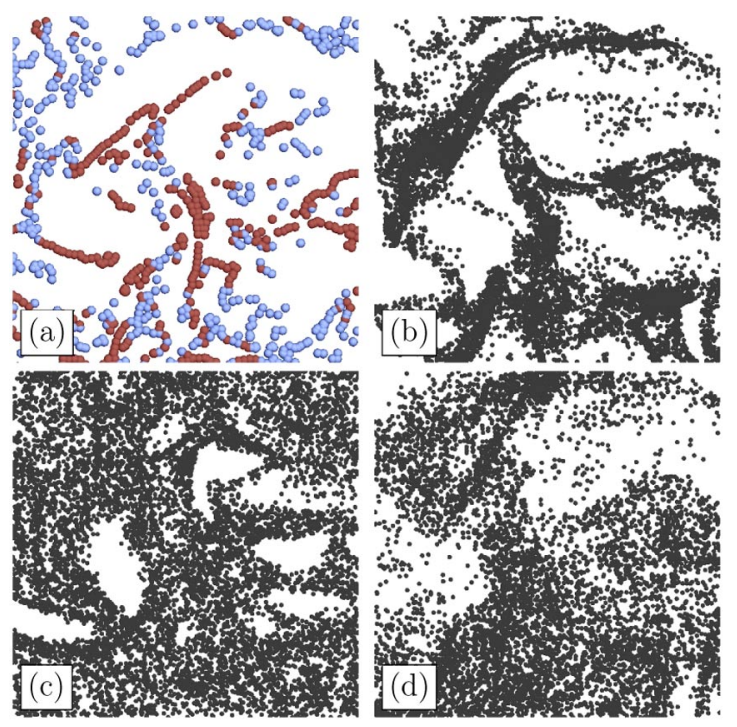

FIG. 4 (color online). (a) Distribution (dark red, $\lambda_{1}>0.2 \tau_{\eta}^{-2}$; light blue, $\lambda_{1}<0.2 \tau_{\eta}^{-2}$; the threshold $0.2 \tau_{\eta}^{-2}$ is chosen so that (4) is satisfied for $\mathrm{St}=2$ at $\lambda_{1}$ equal to that threshold.) of points in $\mathcal{A}$ in a square plane of size $(140 \eta)^{2}$. (b)-(d) Particle distribution in a thin (thickness is $5 \eta$ ) layer in the same location as (a). $\mathrm{St}=2$ (b), 0.1 (c), and 5 (d).

voids associated with points in $\mathcal{A}$ where $\lambda_{1}$ is large [dark red points in Fig. 4(a)] survive. Of course, if we further decrease the Stokes number, then the number of points which satisfy (4) decreases further, and the cluster of particles thickens further too. In such a case, which corresponds to the limit where particles are effectively fluid particles, the clustering cannot be brought about by the sweep-stick mechanism. On the other hand, if we increase the Stokes number [see Fig. 4(d) for $\mathrm{St}=5$ ], particles overshoot the structure $\mathcal{A}$, and the particle cluster becomes faint compared to smaller St cases. Generally, larger values of $\lambda_{1}$ (i.e., larger acceleration gradients) are created by smaller-scale structures. Therefore, the particle voids which are clear in cases of smaller St loose their definition for large St, and only faint clusters accompanied with larger voids survive around smaller $\lambda_{1}$ [light blue points in Fig. 4(a)]. If we further increase St towards values of $\tau_{p}$ larger than the integral time scale, then not only (4) but also (2) are violated. Hence, any clustering present for values of $\tau_{p}$ larger than the integral time scale cannot be accounted for by the sweep-stick mechanism.

The proposed sweep-stick mechanism has been shown to well describe the clustering of heavy small particles in three-dimensional HIT. This mechanism causes particle clusters to reflect the acceleration structure $\mathcal{A}$ defined by (3). This implies that the particle clustering cannot be explained solely in terms of moduli of fluid vorticity, strain rate, pressure gradient or acceleration, but that we need to take into account the topological information of the fluid acceleration field. Furthermore, the clustering condition
(4), which depends on $\tau_{p}$ (particle parameter) and $\lambda_{1}$ (a local property of $\mathcal{A}$ ), is a function of spatial position. Hence, it is not possible, in principle, to describe the detailed structure of the cluster by a spatially averaged quantity such as the local-in-scale Stokes number. It might be worth returning to two-dimensional inverse-energy cascading turbulence in order to show that the explanation given in $[7,9]$ of particle clustering in such flows is consistent with the present explanation because $\boldsymbol{a}=\mathbf{0}$ points are nodes linking $\boldsymbol{a} \cdot \boldsymbol{e}_{1}=0$ lines, and such links are pervasive and effectively define $\mathcal{A}$. Although we have restricted ourselves mainly to the qualitative description of particle clusters in terms of $\mathcal{A}$, the introduction of such a coherent structure should be useful for investigating the statistics or constructing models for impurity and particle distributions in turbulence.

The DNS was carried out on NEC SX-7/160M5 with the support of the NIFS Collaborative Program.

[1] D. Borue, Y. Couder, P. H. J. van Dam, and S. Douady, Phys. Rev. E 47, R28 (1993).

[2] M. Maxey, J. Fluid Mech. 174, 441 (1987).

[3] K.D. Squires and J. K. Eaton, Phys. Fluids A 3, 1169 (1991).

[4] J. K. Eaton and J. R. Fessler, Int. J. Multiphase Flow 20, 169 (1994).

[5] G. Boffetta, F. De Lillo, and A. Gamba, Phys. Fluids 16, L20 (2004).

[6] H. Yoshimoto and S. Goto, J. Fluid Mech. 577, 275 (2007).

[7] S. Goto and J. C. Vassilicos, Phys. Fluids 18, 115103 (2006).

[8] M. Cencini, J. Bec, L. Bifferale, G. Boffetta, A. Celani, A. S. Lanotte, S. Musacchio, and F. Toschi, J. Turbul. 7, N 36 (2006).

[9] L. Chen, S. Goto, and J. C. Vassilicos, J. Fluid Mech. 553, 143 (2006).

[10] J. Bec, L. Biferale, G. Boffetta, A. Celani, M. Cencini, A. Lanotte, S. Musacchio, and F. Toschi, J. Fluid Mech. 550, 349 (2006).

[11] J. Bec, L. Biferale, M. Cencini, A. Lanotte, S. Musacchio, and F. Toschi, Phys. Rev. Lett. 98, 084502 (2007).

[12] M. Maxey and J. Riley, Phys. Fluids 26, 883 (1983).

[13] F. Schwander, E. Hascoet, and J.C. Vassilicos (to be published).

[14] For example, there are less than 100 points in the layer shown in Fig. 1(a). This spareness of $\boldsymbol{a}=\mathbf{0}$ points may be related to the tubular forms of coherent eddies, and thereby to $\boldsymbol{\nabla} p=\mathbf{0}$ points, even though such points do not necessarily coincide with $\boldsymbol{a}=\mathbf{0}$ points. The local extrema of pressure $p$ on the plane perpendicular to a tubular eddy play a role in the clustering, but they are not three-dimensional extrema.

[15] S. Goto, D. R. Osborne, J. C. Vassilicos, and J. D. Haigh, Phys. Rev. E 71, 015301(R) (2005). 